\title{
Encoding of Prospective Tasks in the Human Prefrontal Cortex under Varying Task Loads
}

\author{
Ida Momennejad ${ }^{1,2,3,4,5,6,7}$ and John-Dylan Haynes ${ }^{1,2,3,4,5,6}$ \\ ${ }^{1}$ Bernstein Center for Computational Neuroscience Berlin, Charité-Universitätsmedizin Berlin, 10115 Berlin, Germany, ${ }^{2}$ Berlin School of Mind and Brain, \\ Humboldt Universität zu Berlin, 10099 Berlin, Germany, ${ }^{3}$ Department of Psychology, Technical University Dresden, 01069 Dresden, Germany, ${ }^{4}$ Berlin \\ Center for Advanced Neuroimaging, Charité-Universitätsmedizin Berlin, 10115 Berlin, Germany, ${ }^{5}$ Department of Neurology, Charité-Universitätsmedizin \\ Berlin, 10115 Berlin, Germany, ${ }^{6}$ Department of Psychology, Humboldt Universität zu Berlin, 10099 Berlin, Germany, and ${ }^{7}$ Department of Psychology, \\ Princeton Neuroscience Institute, Princeton University, New Jersey 08544
}

Successful realization of planned actions requires the brain to encode intentions over delays. Previous research has indicated that several regions in the rostral or anterior prefrontal cortex (PFC) encode delayed intentions. However, different processes may encode the same future task depending on task load during the delay. This difference may depend on the computational resources available when the delay is occupied with an ongoing task and when it is task-free. Here we directly investigated and compared the representation of delayed intentions in the human brain in the presence and absence of ongoing task load during the delay. We acquired fMRI data in combination with an event-based prospective memory design where human subjects remembered to perform the same future tasks over occupied and task-free delays. We used time-resolved multivoxel pattern classification and found that: (1) rostrolateral PFC (BA 46) encoded the delayed intention during both delay types; (2) rostromedial PFC (BA 10) encoded the intentions during occupied delays; whereas (3) a variety of more posterior regions, including the anterior cingulate cortex (BA 24), the supplementary motor area (BA 6), and the precuneus, encoded intentions during task-free delays. Overall, the medial PFC encoded delayed intentions more rostrally in the presence of an ongoing delay task and more caudally in its absence. Thus, rostromedial PFC may play a specialized role in the encoding of prospective memory that depends on higher computational demands (e.g., given higher task load during the delay). In contrast, the rostrolateral PFC is a more general area that encodes future intentions regardless of task load.

\section{Introduction}

A pilot needs to perform various checks and adjustments before takeoff. Some checks are performed immediately, whereas others need to be delayed. During some delays, the pilot may be occupied with other checks (e.g., checking the wings); during other delays, she merely waits until the right moment (e.g., until the control tower sends a signal). Regardless of the pilot's task load during the delay, all checks are vital to risk-free takeoff: Dismukes (2008) reported that failing to complete delayed intentions accounts for $\sim 20 \%$ of major aviation accidents. Kliegel and Martin (2003) noted that $50-80 \%$ of everyday memory problems are reported to involve problems with successful realization of delayed intentions (Terry, 1988). In high-risk tasks and everyday

Received Feb. 1, 2013; revised July 31, 2013; accepted Aug. 6, 2013.

Author contributions: I.M. and J.-D.H. designed research; I.M. performed research; I.M. contributed unpublished reagents/analytic tools; I.M. analyzed data; I.M. and J.-D.H. wrote the paper.

This work was supported by the Bernstein Computational Neuroscience Program of the German Federal Ministry of Education and Research BMBF Grant 01GQ0411, the Federal Ministry of Education and Research SFB 940/1, and the Excellency Initiative of the German Federal Ministry of Education and Research DFG Grant GSC86/1-2009. We thank Carlo Reverberi for comments.

The authors declare no competing financial interests.

Correspondence should be addressed to either: Dr. Ida Momennejad or Dr. John-Dylan Haynes, Charité-Universitätsmedizin Berlin, Bernstein Center for Computational Neuroscience, Haus 6, Philippstrasse 13, 10115 Berlin, Germany, E-mail: idam@princeton.edu or haynes@bccn-berlin.de.

DOI:10.1523/JNEUROSCI.0492-13.2013

Copyright $\odot 2013$ the authors $\quad 0270-6474 / 13 / 3317342-08 \$ 15.00 / 0$ life, accomplishing planned action crucially relies on computational brain processes that encode upcoming tasks.

The capacity to remember future tasks while performing distracting activities, and to execute the delayed task at the right time, is referred to as prospective memory (PM) (Einstein and McDaniel, 1990; Brandimonte et al., 1996; Ellis, 1996; Burgess et al., 2011). Recent neuroimaging evidence shows that delayed intentions and PM can be decoded from activity patterns in the anterior or rostral prefrontal cortex. These studies have decoded delayed intentions when participants had no ongoing task during the delays (Haynes et al., 2007), in PM paradigms where delays were occupied with an ongoing task (Gilbert, 2011; Momennejad and Haynes, 2012), and even during unconscious preparation of task-related choices (Soon et al., 2008). These findings confirm the established role of prefrontal cortex (PFC) in the encoding and organization of planned actions in paradigms of executive function involving branching, task-switching, task set preparation, abstraction, hierarchical action selection, and PM (Koechlin et al., 1999; Miller and Cohen, 2001; Sakai and Passingham, 2003; Ramnani and Owen, 2004; Rushworth et al., 2004; Simons et al., 2006; Koechlin and Hyafil, 2007; Badre, 2008; Sakai, 2008).

An important question that remains unanswered is whether and how the encoding of intentions depend on the availability of computational resources during the delay period. One way to address this question is to vary the nature of ongoing activity. So far it remains unclear how the brain encodes future intentions when the 
delay is occupied with ongoing tasks and when it is task-free. Here we directly investigated how the human brain encodes future intentions depending on task load during the delay.

We performed an fMRI study where participants formed, maintained, and executed future intentions under two different delay conditions: (1) during occupied delays, participants held their future intentions in mind while performing an ongoing task; and (2) during task-free delays, participants did not engage in an ongoing activity and focused on the upcoming task. Using multivoxel pattern classification, we could decode delayed intentions from rostrolateral PFC (RLPFC) during both delays, from rostromedial PFC (RMPFC) during occupied delays, and from anterior cingulate cortex (ACC), SMA, and the precuneus during task-free delays. We found that encoding delayed intentions recruited RLPFC in general, and RMPFC under higher computational demand (e.g., when less computational resources were available due to higher task load).

\section{Materials and Methods}

Participants

Twenty-three healthy volunteers participated in the study (10 female). No subject reported a history of medical or mental hospitalization. All participants signed a written consent to participate in accordance with the guidelines of the local ethics committee (Charité-Universitätsmedizin Berlin).

\section{Stimuli and experimental design}

All stimuli were created with the Cogent 2000 toolbox (Laboratory of Neurobiology, University College London; http://www.vislab.ucl.ac. uk/Cogent/) implemented in MATLAB (The MathWorks) on a Windows computer. During the fMRI session, the stimuli were projected on a screen behind the MRI scanner and viewed via a mirror attached to the scanner head coil. Throughout the experiment, participants indicated their behavioral responses with a $2 \times 2$ button box, with two buttons under the index and middle fingers of each hand, respectively. All stimuli were presented with white on black background during the experiment but are displayed as black on white in Figures 2 and 3 for clarity.

We used a $2 \times 2$ factorial experimental paradigm where participants remembered either of two future tasks over two delay types (occupied or task-free delays). All tasks involved an instruction, a delay period, and the realization of a delayed intention. At instruction, two visual symbols were presented above and below fixation (Fig. 1). The symbol above fixation indicated delay type (i.e., whether the delay period was filled with an ongoing task or not) (Fig. 1B). If this symbol indicated that the delay period was occupied with an ongoing task, participants performed a third task (i.e., color judgment) during the delay. If the symbol indicated a task-free delay, participants were instructed to focus on the prospective task and ignore the stream of stimuli during the delay.

The symbol below fixation indicated the future task, or what to do after the delay (i.e., parity or magnitude judgment) (Fig. 1B). To ensure that holding the delayed task in mind was not confounded with holding a specific instruction symbol, we used two instruction symbols for each task (Fig. 1B). The symbols were intuitive and visually distinct; this ensured that the encoding of the same task could be studied by training and testing the classifier on different visual cues. When the lower instruction symbol indicated either of the two parity symbols (Fig. $1 B$ ), participants had to remember to perform the parity task (i.e., judge whether numbers were odd or even). When the lower instruction symbol indicated either of the two magnitude symbols (Fig. $1 B$ ), participants had to remember to perform the magnitude task (i.e., judge whether numbers were bigger or smaller than 5).

The delay period immediately followed the instruction and lasted $8-12$ s (4-8 stimuli for the color task). During the delay, a constant flow of color stimuli appeared on the screen (Fig. 1C). The rate of stimulus presentation was constant at $0.5 \mathrm{~Hz}$. The stimuli included a red or green circle above fixation and two response symbols below fixation (Fig. 1C). The response symbols corresponded to "red" or "green" and appeared on

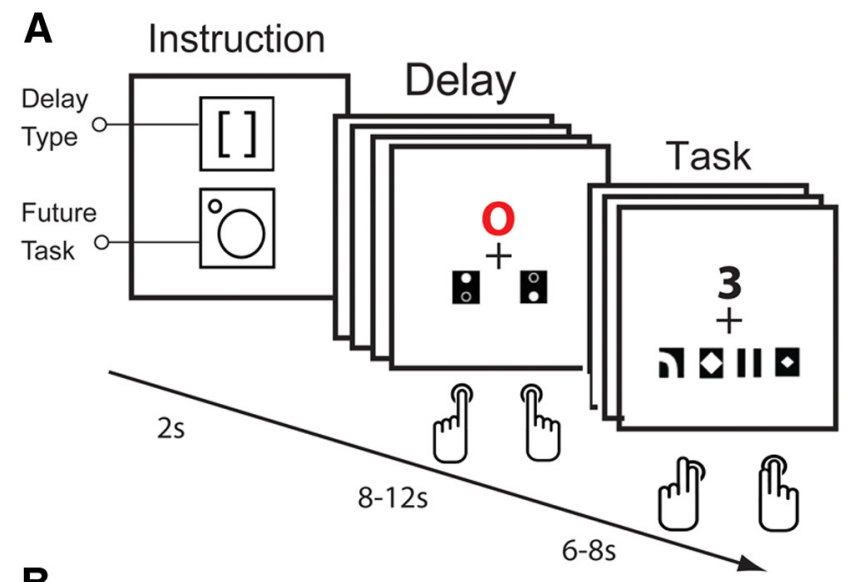

B
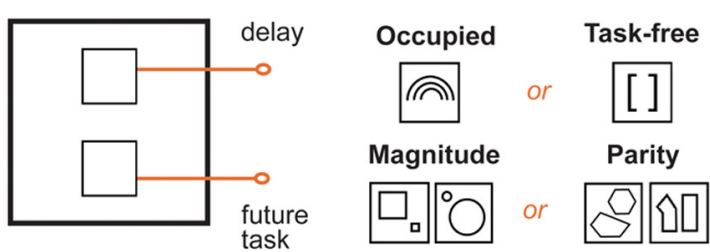

C

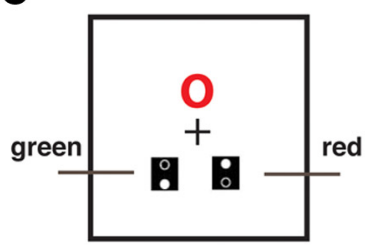

D

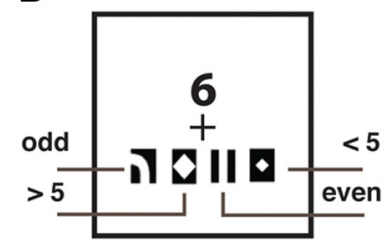

Figure 1. Experimental design. We used a $2 \times 2$ design with two delay conditions and two future tasks $(\boldsymbol{A})$. At the beginning of each trial, an instruction screen $(\boldsymbol{B})$ appeared where the symbol above fixation indicated the delay type (occupied or task-free delay) and the symbol below fixation indicated the future task (parity or magnitude, $\boldsymbol{B}$ ). This was immediately followed by the delay stimuli; a stream of red or green circles and two response symbols ( $\boldsymbol{C}$. To respond, participants indicated the randomized location of the corresponding response symbol via button presses $(\boldsymbol{A})$. Given an occupied delay instruction, participants performed the color task during this phase; whereas given a task-free delay instruction, they did not perform any task and passively observed the delay stimuli until the end of the delay. The delay was followed by the task execution phase where a stream of single-digit number stimuli between 1 and 9 (not 5) appeared (D). Participants performed the delayed task on these stimuli: Parity: is the number odd or even? Magnitude: is the number bigger or smaller than 5 ? All response symbols were displayed in a pseudorandomized order, and responses were uncorrelated with specific locations on screen. We acquired fMRI data while participants performed 6 runs of 24 such trials inside the scanner.

the left or the right side in a pseudorandom fashion. Therefore, the responses were not confounded with their location on screen. Participants only performed the color judgment task on the stimuli if the instruction indicated an occupied delay. If the instruction indicated a taskfree delay, they merely held the delayed intention in mind.

To report their judgments during occupied delays, participants pressed their left or right index finger corresponding to the location of the symbol that indicated their response. Importantly, the pseudorandom allocation of the response symbols to left and right ensured that no specific color response was confounded with left or right button presses. At the end of the delay, the fixation-cross changed its color from white to green for $500 \mathrm{~ms}$. Participants were instructed to recall and execute the PM task after the switch cue.

During the task phase, a constant flow of numbers appeared on the screen for $6-8 \mathrm{~s}$ ( 3 or 4 stimuli) at a rate of $2 \mathrm{~s}$ per stimulus. The stimuli consisted of a single-digit number (other than 5 ) above fixation and four response symbols below (Fig. 1D). Two of 4 response symbols corresponded to the parity task: one indicated "odd" and the other "even." The other two response symbols corresponded to the magnitude task: one 
indicated "bigger than 5" and the other "smaller than 5" (Fig. 1D). Participants selected the response symbol that indicated their response by pressing one of four response buttons (two buttons on the index and the middle finger on both left and right hands; Fig. 1A). These buttons, respectively, corresponded to the temporary location of the response symbols on the screen. Importantly, all possible response symbols were present on every stimulus; therefore, visual differences did not remind the participant of the instructed task, and they had to retrieve the task from memory. Participants did not receive any feedback throughout the runs.

\section{Training}

Participants underwent two sessions of training in which they learned all instruction and response symbols and practiced performing all tasks outside the scanner. The duration of the first session was $1 \mathrm{~h}$, and it took place one day before scanning. During the first training session, participants familiarized themselves with the instruction and task symbols and learned the experimental procedure. The training was divided into sections that adaptively trained participants to ensure they performed all tasks equally well. The second training session lasted $8 \mathrm{~min}$ and took place just before entering the scanner. This session allowed participants to refresh their memory and practice an entire experimental run.

\section{fMRI acquisition}

Functional and structural images were acquired using a 3-Tesla Siemens Trio MRI scanner with a 12-channel head coil at the Berlin Center for Advanced Neuroimaging. Each participant was measured during 6 experimental sessions and 2 rest sessions using an echo-planar-imaging sequence with a repetition time of $2070 \mathrm{~ms}$ and echo time of $30 \mathrm{~ms}$, an in-plane $64 \times 64$ matrix of 34 slices with $3 \mathrm{~mm}$ slice thickness and $0.5 \mathrm{~mm}$ interslice gap, sampling field of view of $192 \times 192 \mathrm{~mm}^{2}$, flip angle of $90^{\circ}$, and an anterior to posterior phase encoding direction. T1-weighted images and field maps were acquired using separate sequences (T1weighted MPRAGE: 192 sagittal slices, repetition time $=1900 \mathrm{~ms}$, echo time $=2.52 \mathrm{~ms}$, flip angle $=9^{\circ}$, field of view $=256 \mathrm{~mm}$, resolution: $256 \times 256 \times 192$ ). Separate phase and magnitude field map images were acquired, analyzed, and used in the preprocessing pipeline to optimize the correction of image distortions.

\section{Data analysis}

fMRI preprocessing. Before the multivariate decoding analysis, neuroimaging data were analyzed using statistical parametric mapping methods implemented in the SPM 8 in MATLAB (http://www.fil.ion.ucl.ac. $\mathrm{uk} / \mathrm{spm} / \mathrm{software} / \mathrm{spm} 8 /$ ). For every subject and every experimental run, all volumes were realigned to the first volume of the first run and then corrected for different timing in slice acquisition. The resulting images were then unwarped using field maps. The field maps were calculated based on phase and magnitude images acquired after each participant completed the experiment in the scanner. The field maps allowed us to correct for geometric distortions caused by magnetic field inhomogeneities (Hutton et al., 2002). This further correction was especially effective to optimize normalization at the margins of the brain, particularly the anterior PFC where distortions are strong. As a result of the preprocessing steps, all functional images were realigned, slice-time corrected, coregistered to the anatomical image, unwarped, and normalized to MNI standard space with a voxel size of $3 \times 3 \times 3 \mathrm{~mm}$.

Finite impulse response general linear model. To model the fMRI time series with the highest affordable temporal resolution and with as little assumptions regarding the shape of the signal as possible, we used a general linear model (Friston et al., 1994) with a finite impulse response basis function (Henson, 2004). The finite impulse regressor modeled the BOLD response separately for each time bin (Henson et al., 2001), corresponding to each full scanned volume of the brain (every $2.07 \mathrm{~s}$ ) in the time series. For each condition, the BOLD response was decomposed into 10 stick functions of $2.07 \mathrm{~s}$ width (corresponding to the repetition time or the time it takes to acquire a full brain volume) and 20.07 s length. We estimated regressors for four conditions: (1) storing parity over taskfree delays, (2) storing parity over occupied delays, (3) storing magnitude over task-free delays, and (4) storing magnitude over occupied delays. We included all conditions in one general linear model.
Time-resolved searchlight decoding. To identify regions that store delayed intentions during task-free and occupied delays, we used a moving searchlight pattern analysis (Kriegeskorte et al., 2006; Haynes et al., 2007). The following steps were repeated separately for each subject $S$, every time bin $\mathrm{T}$, and every condition (occupied and task-free) of the resulting parameter estimates of the finite impulse response analysis (Soon et al., 2008; Bode and Haynes, 2009; Momennejad and Haynes, 2012).

We assessed the decodable local pattern information at each position in the brain as follows. For each voxel $\mathrm{V}$ in the brain, we extracted the parameter estimates within a spherical cluster of surrounding voxels (radius $=4$ voxels). The parameter estimates were extracted for the two delayed intentions and each run separately, resulting in 12 pattern vectors for each of the 10 time bins ( 2 conditions $\times 6$ runs $\times 10$ time bins). The length of each vector corresponded to the number of voxels in the searchlight $(N=251)$. Each vector was labeled according to its corresponding delayed intention. These pattern vectors were used as training and test data in decoding. A leave-one-out approach with sixfold crossvalidation was used to calculate the overall accuracy of intention classification (Momennejad and Haynes, 2012).

On every step of the sixfold cross-validation, pattern vectors from one run were left out as test data, whereas the pattern vectors of the remaining 5 test-runs were used as training set. The training and test data were then fed into a linear support vector classifier $($ SVC, $c=1)$ for multivariate pattern analysis. The support vector classifier learned to classify the task that the participant held in mind (i.e., parity vs magnitude). The learned classification parameters that were estimated for the training data were then applied to the independent test dataset to predict which task the participant held in mind during the delay. The decoding accuracy for each cross-validation step was calculated as the percentage of correctly predicted labels. The cross-validation procedure was repeated 6 times, each time a different run was left out as test data. Finally, the mean of the resulting 6 accuracy values was written to the central voxel of the cluster in the final accuracy map as the decoding accuracy of that voxel.

The results of the time-resolved decoding were 2 sets (for occupied/ task-free delay conditions) of 10 accuracy maps (corresponding to the 10 finite impulse response time bins). The accuracy maps of each participant were smoothed with an $8 \mathrm{~mm}$ FWHM Gaussian kernel. We then entered smoothed accuracy maps of all participants into a one-way ANOVA with factor time and used $t$-contrasts over time bins $2-4$ during the delay period to determine regions that store delayed intentions across delays (see Results; Fig. 3; Tables 1 and 2). The minimum duration of the delay period was 4 trials, corresponding to 4 time bins, and the last trial included the switch cue. Therefore, we used a $t$-contrast on the first 3 time bins of the delay period to avoid including retrieval processes in the second level analysis during the delay (time bins 3-5). We corrected the results for multiple comparison using familywise error (FWE) correction $(p<0.05)$ at cluster level with height threshold of $p<0.005$ (Friston et al., 1996).

Cross-condition analysis. To investigate patterns that similarly encode prospective intentions regardless of delay type, we conducted two crosscondition decoding analyses. In each analysis, we trained a linear SVC to classify future task during one type of delay and used the classification parameters to decode the future task in trials with the other delay type. Specifically, in the first analysis for each subject, we trained the SVC (to classify parity vs magnitude as future task) on all data during occupied delays and used the SVC parameters to predict the identity of the future task in independent trials with task-free delays. In the second analysis, we trained the classifier on data from task-free delays to decode the future task during occupied delays. For group level analysis of decoding results during each delay type, we used one-way ANOVAs (factor time, 7 levels: time bins 1-7) on the decoding results of 23 subjects (FWE corrected at cluster level, $p<0.05$ ).

Direct comparison of task decoding during occupied versus task-free delays. For a whole-brain direct comparison of findings across the two delay types, we used a two-way ANOVA with factors delay type (two levels: occupied and task-free) and time (four levels: time bins 3-6). Using T-contrasts over the 3 time bins of the delay period (time bins 3-5), we asked which regions had significantly more information about the future 
Table 1. Decoding delayed intentions under varying task load ${ }^{a}$

\begin{tabular}{|c|c|c|c|c|c|}
\hline$x$ & $y$ & $z$ & $t$ value & z score & Region \\
\hline \multicolumn{6}{|c|}{ Decoding PM tasks in occupied delays (cluster FWE, $p<0.05$ ) } \\
\hline-9 & 65 & 28 & $4.48^{* *}$ & 4.65 & BA 10, dorsal RMPFC \\
\hline-15 & 53 & 2 & $4.29 * *$ & 4.19 & BA 10, RMPFC \\
\hline-9 & 65 & -6 & $4.25^{* *}$ & 4.16 & BA 11, ventral RMPFC (includes BA 10) \\
\hline \multicolumn{6}{|c|}{ Decoding PM tasks in task-free delays (cluster FWE, $p<0.05$ ) } \\
\hline 21 & -88 & 19 & $4.23^{* *}$ & 4.14 & BA 18 , superior occipital lobe \\
\hline 21 & -79 & -17 & $3.63^{* *}$ & 3.57 & Right cerebellum \\
\hline-3 & -7 & 76 & $3.51^{*}$ & 3.46 & BA 6 \\
\hline-9 & -7 & 79 & $4.21^{* *}$ & 4.12 & BA 6, SMA (analysis: bins 3 and 4, TF) \\
\hline 30 & 41 & 7 & $3.18^{*}$ & 3.14 & BA $24, A C C$ \\
\hline 9 & -1 & 40 & $3.80^{* *}$ & 3.73 & BA 24, ACC (analysis: bins 3 and 4, TF) \\
\hline \multicolumn{6}{|c|}{ Overlapping regions with task information in occupied and task-free delays } \\
\hline 39 & 62 & 19 & 2.93 & 3.54 & Conjunction: BA 46, right RLPFC \\
\hline 39 & 53 & 19 & 2.64 & 2.62 & Conjunction: BA 46 \\
\hline 33 & 44 & 19 & 2.63 & 2.60 & Conjunction: BA 46 \\
\hline
\end{tabular}

${ }^{a}$ Regions with significant decoding of delayed intentions during occupied delays, task-free delays, and conjunction analysis. Reported coordinates represent voxels with peak decoding accuracy in each cluster. Decoding results marked with no asterisks overlapped in occupied and task-free delays and were FWE corrected at cluster level ( $p<0.05)$ using small volume correction. The volume of interest was defined with a radius of $20 \mathrm{~mm}$. All coordinates are reported in MNI space. ${ }^{*} t$ values passed FWE correction in a T-contrast over time bins 3 and 4 and were found in the present analysis (T-contrasts over bins $3-5$ ) using a lower threshold.

${ }^{* *} t$ values are FEW-corrected for multiple comparisons at cluster level $(p<0.05)$ with a height threshold of $(p<0.005)$.

Table 2. A priori ROls with future task information ${ }^{a}$

\begin{tabular}{|c|c|c|c|c|c|c|}
\hline Region & $x$ & $y$ & $Z$ & $t$ value & z score & Study \\
\hline BA 32 & 3 & 42 & 15 & $4.48^{* *}$ & 4.38 & Haynes et al., 2007 (task-free delay, self-initiated recall) \\
\hline BA 10 & 0 & 60 & -3 & $4.29^{* *}$ & 4.19 & Soon et al., 2008 (occupied delay, self-initiated recall) \\
\hline BA 10 & 9 & 62 & 4 & $3.02 *$ & 2.99 & Gilbert, 2011 (event-based PM, occupied delay, cued recall) \\
\hline BA 10 & 3 & 60 & 39 & $4.48^{* *}$ & 4.38 & Momennejad and Haynes, 2012 (time-based PM, occupied delay, self-initiated recall) \\
\hline
\end{tabular}

${ }^{a}$ We defined a priori ROIs on the basis of reported peaks from four previous decoding studies on delayed intentions. We found that, during occupied delays, all ROls contained significant information regarding the future task. For a cortical illustration of these regions, see Figure 5.

*Corrected for multiple comparisons using small volume correction with a radius of $20 \mathrm{~mm}$.

**FEW-corrected at cluster level $(p<0.05)$.

task during one type of delay versus the other. Contrast occupied $>$ task-free investigated regions with significantly higher accuracies during these 3 time bins in occupied delays, and the contrast task-free $>$ occupied investigated the reverse. All results were corrected for multiple comparisons at cluster level ( $n=23$, FWE corrected at cluster level, $p<0.05)$.

Conjunction analysis. To identify any overlapping regions between the two conditions, we conducted a further conjunction analysis. We identified the voxelwise intersection between the second-level accuracy maps for occupied and task-free analyses (i.e., an AND operation).

\section{Results}

\section{Behavioral results}

We first analyzed the reaction times (RTs) and accuracy of responses to the ongoing task (i.e., the color task). There were no significant effects of the prospective task (i.e., parity or magnitude) on the RTs $\left(t_{(22)}=1.16, p>0.05\right.$; Fig. 2$)$ or the accuracy of responses $\left(t_{(22)}=1.21, p>0.05\right.$; Fig. 2 right $)$ to the color task. In short, we did not find any evidence that the specific task that participants held in mind influenced behavioral performance on the ongoing task.

In a further step, we analyzed the behavioral responses to the prospective tasks using a two-way repeated-measures ANOVA with factors, prospective task (two levels: parity and magnitude) and delay type (two levels: occupied and task-free). Overall we did not observe any main effects of prospective task, delay type, or their interaction on prospective task performance. Specifically, we found no main effect of prospective task on RTs $\left(F_{(1,22)}=\right.$ $0.073, p=0.78)$ or accuracy of responses $\left(F_{(1,22)}=0.48, p=\right.$ $0.48)$; no main effect of the delay type on either RTs $\left(F_{(1,22)}=\right.$ $0.005, p=0.94)$ or accuracy of responses to the prospective task $\left(F_{(1,22)}<0.001, p=0.99\right)$; and no interaction effects of prospec-
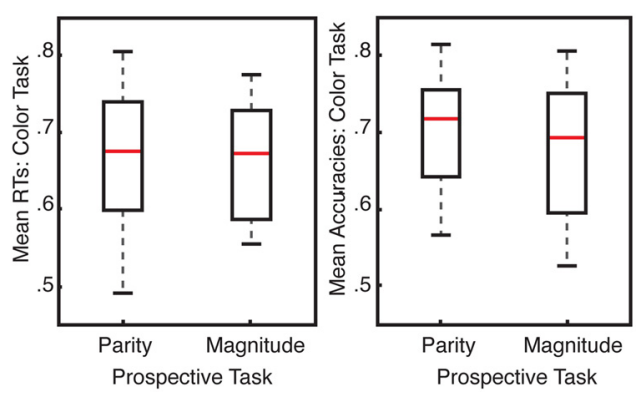

Figure 2. The effect of delayed intentions on ongoing task performance. There was no significant effect of the future intention (i.e., parity vs magnitude) on the RTs $\left(t_{(22)}=1.16, p>\right.$ $0.05)$ or accuracy of responses $\left(t_{(22)}=1.21, p>0.05\right)$ to the color task during occupied delays.

tive task $\times$ delay type on $\operatorname{RTs}\left(F_{(1,22)}=0.001, p=0.96\right)$ or accuracy of responses $\left(F_{(1,22)}<0.001, p=0.99\right)$.

\section{Neuroimaging results}

Brain regions, coordinates, and statistics corresponding to the neuroimaging results are displayed in Figures 3, 4, and 5 and Tables 1 and 2. A conjunction analysis revealed that the rostrolateral PFC (BA 46) encoded prospective intentions over both occupied and task-free delays (Table 1). During occupied delays, we could decode the delayed intention from broad regions of BA 10 , stretching from the dorsomedial to ventromedial aspects of the rostral PFC into BA 11 (Figs. 3 and 4). These regions were located in the most anterior parts of the rostral PFC and stretched back to the rostral ACC (BA 32). We further looked for significant decoding in regions around the cluster found in the con- 

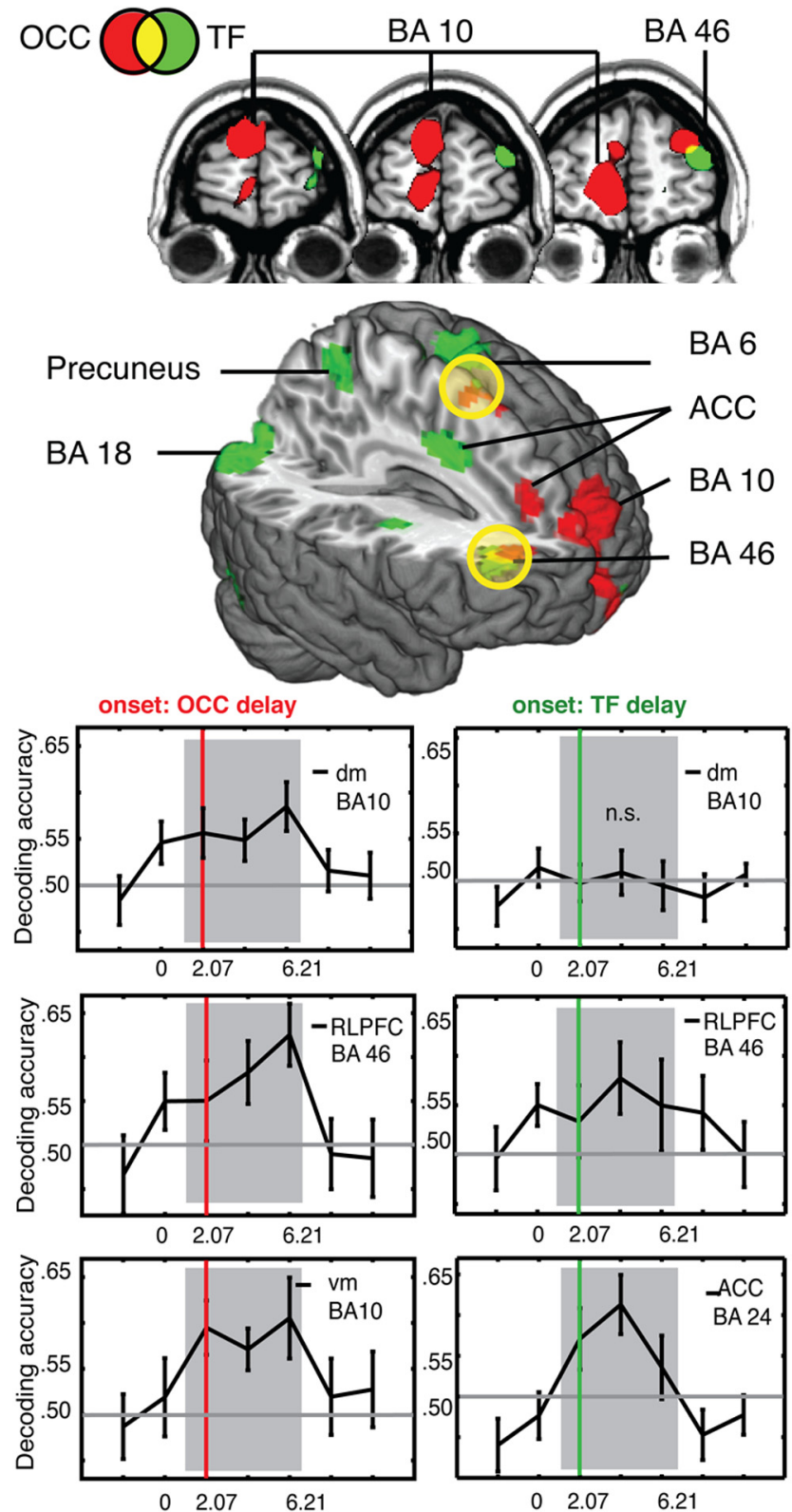

Seconds

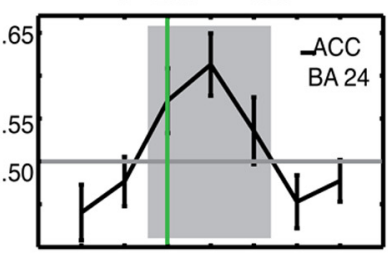

$\begin{array}{lll}0 & 2.07 & 6.21\end{array}$

Seconds

Figure 3. Time-resolved decoding of delayed intentions during task-free versus occupied delays. Top, Regions with significant decoding of prospective task (parity vs magnitude) during the delay. Yellow circles represent the results of cross-condition decoding analysis: the RLPFC and the SMA. All results were corrected for multiple comparisons but are here displayed at $p<$ 0.005 uncorrected height threshold for illustration. For corrected $p$ values, see Table 1. Bottom, Time course of decoding accuracy in regions that store delayed intentions. Gray boxes represent the time bins that were used on the second-level $t$-contrast in the ANOVA. The time series was shifted by 2 time bins to account for the delay in the hemodynamic response function. The red lines in the left-hand plots indicate the onset of occupied delays. The green lines in the righthand plots indicate the onset of task-free delays. The coordinates of the specified regions are as follows: dorsomedial (dm) BA 10, -9, 65, 28; ventromedial (vm) BA 10, -9, 65, -6; RLPFC (right BA 46), 39, 62, 19; ACC (BA 24), 9, -1, 40; SMA (BA 6), -9, -7, 79. 0CC, Occupied delay; TF, task-free delay.

junction analysis. We found that, during occupied delays, the overlapping cluster extended to more anterior regions (BA 10).

During task-free delays, we found that regions in the precuneus, the occipital lobe, ACC (BA 24), lateral BA 6, and the SMA (BA 6) stored the prospective intention (Fig. 3; Table 1). We also looked for significant decoding in regions around the cluster

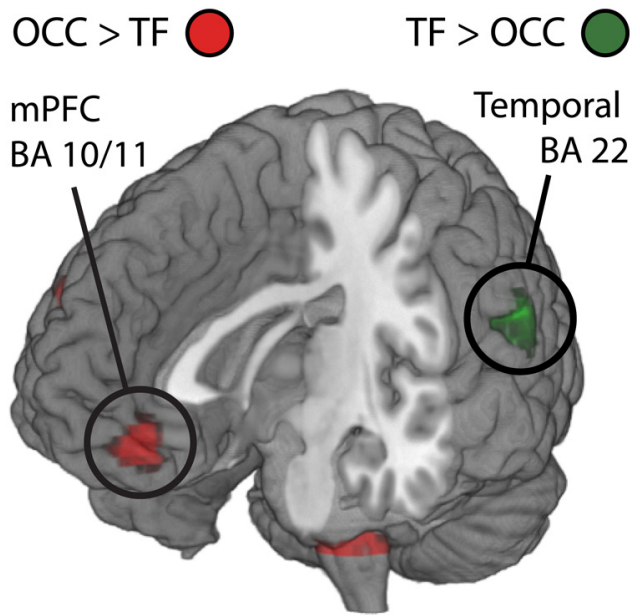

Figure 4. Direct comparison between the decoding of future task during occupied (OCC) versus task-free (TF) delays. A two-way ANOVA ( 2 delay type $\times 4$ time bins) was used to directly compare decoding analyses of occupied (OCC) and task-free (TF) delay conditions. T-contrasts were applied to the three time bins of the delay period. In the $0 \mathrm{CC}>\mathrm{TF}$ contrast, the encoding of the future task was significantly higher medial PFC (BA 10/11, peak at MNI coordinate $[3,41$, $-11]$, FWE corrected at $p<0.05$ ). In the TF $>0 C($ contrast, decoding of the future task was significantly higher in the temporal cortex (BA 21/22, peak at $[-41,-49,19]$, FWE corrected at cluster level, $p<0.05$ ).

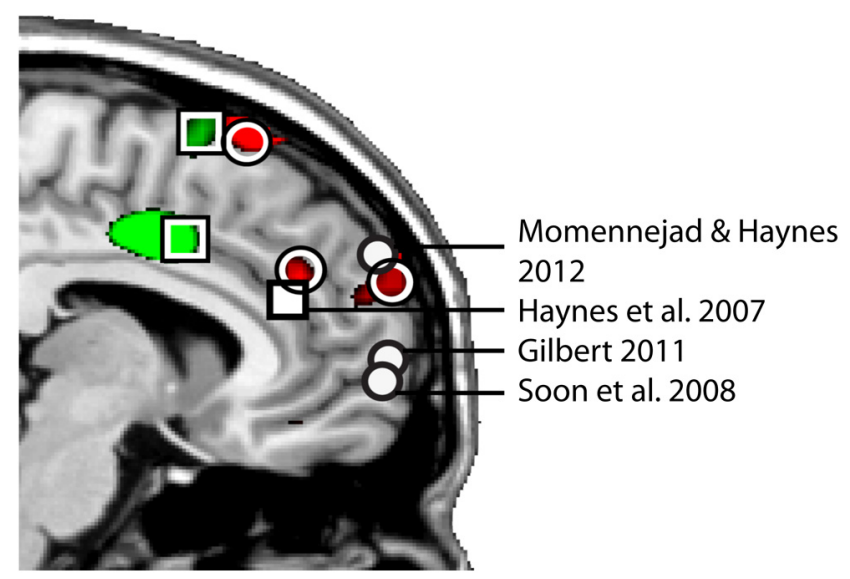

Figure 5. The role of medial PFC in storing delayed intentions. Regions that were reported in this and other neuroimaging studies of delayed intentions are displayed on the medial wall of the PFC. The reported peaks were slightly moved to fit on the same medial slice for illustration purposes; the precise coordinates are reported in Table 2 . Hollow shapes indicate the results of the present study where patterns of decoding over occupied delays are displayed in red and those of task-free delays in green. Filled shapes indicate regions that stored intention information in previous decoding studies. Regions marked by squares indicate decoding of delayed intentions over task-free delays. Both experimental designs used cued recall (Haynes et al., 2007; present study). Regions marked by circles indicate decoding results of delayed intentions over occupied delays in both event-based PM paradigms that used cued recall (Gilbert, 2011; present study), in a time-based PM paradigm that used self-initiated recall (Momennejad and Haynes, 2012), and in a delayed choice paradigm with self-initiated recall (Soon et al., 2008).

found in the conjunction analysis. We found that, during taskfree delays, the overlapping RLPFC cluster extended to more ventral/inferior and caudal PFC regions (BA 47).

Furthermore, we conducted a cross-condition classification analysis to investigate regions that encoded the future task with similar patterns across occupied and task-free delays. Using this cross-condition analysis, we first trained a support vector classifier on the future task during occupied delays and tested the classifier on the future task during task-free delays. Group-level 
analysis of these findings across 23 subjects revealed the right RLPFC (MNI peak at $[33,56,28], k=47, \mathrm{BA} 46$ ) (FWE corrected at $p<0.05$, height threshold at $p<0.005)$, the SMA (MNI $[-13$, $-10,70], k=138, \mathrm{BA} 6$ ), and the left superior temporal cortex (MNI $[-57,-10,4], k=141, \mathrm{BA} 48)$. Second, training a classifier on data from task-free delays and testing the classifier on data from occupied delays revealed regions with future task information under both conditions in the SMA (MNI $[3,-4,67], k=42$, BA 6) and in the left superior temporal cortex (MNI [-63, -21 , 22 ], $k=42$, BA 48). The findings of the two analyses are highlighted in Figure 3 with transparent yellow circles indicating the right RLPFC and the SMA. Together, cross-condition decoding revealed that only the lateral regions of rostral PFC encoded future tasks regardless of delay type, whereas cross-condition decoding in the medial PFC was only possible in more posterior regions.

In a further step, we conducted a two-way ANOVA (delay type $\times$ time) to directly compare the results of decoding the future task during occupied and task-free delays (T-contrasts over time bins 3-5). The occupied $>$ task-free contrast revealed that the medial PFC encoded the future task during occupied but not task-free delays (BA 10/11, peak at MNI coordinate [3, 41, -11 ], FWE corrected at $p<0.05)$. In the task-free $>$ occupied contrast, significantly higher decoding of the future task was observed in a mid temporal region (BA 21/22, peak at $[-41,-49$, 19], FWE corrected at cluster level, $p<0.05$; Fig. 4).

We also created a map that directly compares present findings with those of previous decoding studies (Fig. 5). We first extracted MNI coordinates of voxels with peak decoding accuracy in previous studies to define a priori regions (Haynes et al., 2007; Soon et al., 2008; Gilbert, 2011; Momennejad and Haynes, 2012). We then looked for any significant information in the clusters surrounding these regions using small volume correction with a radius of 20 voxels. We found that during occupied delays all regions passed the multiple comparison correction at cluster level $p<0.05$ (Fig. 5).

In a more stringent analysis step, we separately compared mean decoding accuracies during occupied versus task-free delays for searchlights $(r=4)$ surrounding each of the four a priori peak voxels. Under this more stringent comparison, we observed a significant difference of mean decoding accuracies during occupied versus task-free delays $\left(t_{(22)}=2.21, p=0.03\right)$ in one of the ROIs (MNI coordinate of peak voxel $[0,60,-3]$ ) (Soon et al., 2008). This region was anatomically most proximal to the results of the direct comparison found in the two-way ANOVA described above (Figs. 4 and 5).

\section{Discussion}

The successful realization of delayed intentions, such as buying milk after work, making a grant deadline, or checking an airplane's wing function before take-off, relies on prospective memory (PM). This is the capacity to store and shield intentions across long time delays, often while performing distracting activities, and to remember to execute the intended task once the conditions are met (Einstein and McDaniel, 1990; Brandimonte et al., 1996; Ellis, 1996; Burgess et al., 2011). To our knowledge, no study had directly compared the processes that encode future intentions during delays with and without an ongoing task. Here we used a paradigm that isolates the encoding of delayed intentions depending on the nature of the delay. We directly investigated delay-specific encoding of future task sets in the entire brain and tested the specific role of the RMPFC. We found the following: (1) In both occupied and task-free delays, the future task could be decoded from the right RLPFC (BA 46). (2) During occupied delays, delayed intentions could be decoded from the most anterior parts of the RMPFC (dorsally BA 10, ventrally BA 10 and 11) and the rostral ACC (BA 32). (3) During task-free delays, the future task could be decoded from a variety of more posterior regions, including the caudal medial PFC (ACC/BA 24, BA 6) and the precuneus. Our findings suggest that the rostrocaudal gradient of the medial PFC may play a specialized role in the encoding of future intentions and PM that depends on the architecture of intentional action, such as the nature of the delay period.

A number of previous studies suggest that activation in the anterior or rostral PFC underlies the neural organization of planned actions in executive function (Fuster, 2000; Ramnani and Owen, 2004), including task set preparation and decisions (Sakai and Passingham, 2003; Rushworth et al., 2004; Sakai, 2008), task switching, branching, and coordination of multiple tasks (Koechlin et al., 1999; Braver and Bongiolatti, 2002; Koechlin and Hyafil, 2007), abstraction of task sets (Christoff et al., 2009), hierarchically organized action (Badre, 2008), and PM (Okuda et al., 1998; Simons et al., 2006; Burgess et al., 2011). It has been shown that univariate BOLD activation in RLPFC (lateral BA 10) plays an important role in event-based PM (Simons et al., 2006; Reynolds et al., 2009; Benoit et al., 2012), time-based PM (Okuda et al., 2007), and the comparison of time based and event-based PM (Burgess et al., 2003, 2011; Gilbert et al., 2005, 2006, 2009). Furthermore, lesions to these regions impair both time-based and event-based PM capacities (Burgess et al., 2000; McFarland and Glisky, 2009; Volle et al., 2011). However, univariate methods do not reveal whether these brain regions encode information regarding the content of delayed intentions across delays.

Evidence from recent neuroimaging studies that use multivariate decoding suggests that delayed intentions and PM content can be decoded from activity patterns in the anterior or rostral PFC. These studies have decoded delayed intentions that were freely chosen and maintained across task-free delays (Haynes et al., 2007), free intentions during unconscious preparation for binary choices (Soon et al., 2008), as well as intentions that were instructed and maintained over occupied delays with cued retrieval (Gilbert, 2011) and with self-initiated retrieval (Momennejad and Haynes, 2012). Notably, these findings confirm the aforementioned role of the rostral PFC in the encoding and organization of planned actions. A discussion of the present novel findings follows.

\section{Computational demand and the rostrocaudal gradient of medial PFC}

In the present study, the same future task sets were encoded in more rostral medial regions (BA 10, BA 11, BA 32) during occupied delays and in more caudal medial regions (BA 6, BA 24) during task-free delays. Furthermore, we conducted an a priori ROI analysis in RMPFC (where ROIs were defined based on previous decoding studies) and found that these regions encoded intention information during occupied but not during task-free delays (Table 2; Fig. 5). Overall, we found that, when the task load during the delay period is higher, more rostral medial regions encode the future intention; and when the task load is lower, more caudal medial regions store the upcoming task set. The computational explanation of this observation may be that high task load during the delay period modulates the level of attentional or control processes required to efficiently encode and maintain a future intention (McDaniel and Einstein, 2000). Fur- 
thermore, efficient encoding may require further abstraction of future task information as other computational resources are allocated to ongoing task performance (Rushworth et al., 2004; Christoff et al., 2009). Thus, our findings suggest that encoding future intentions under higher levels of computational demand (which may depend on higher demands for attentional focus or control or abstraction) recruits progressively more dorsal and anterior regions of the medial PFC. This explanation is in line with the architectonic organization of primate PFC and confirms the role of medial PFC in conflict resolution and volition (Miller and Cohen, 2001; Nachev et al., 2005; Petrides, 2005; Isoda and Hikosaka, 2007) and the functional topography of dorsomedial PFC for strategic control of action (Venkatraman et al., 2009; c.f. Shenhav et al., 2013). Together, the specialized recruitment of medial PFC may depend on the level of control required to encode future intentions depending on the availability of computational resources (e.g., given long delays, high task load during the delay, endogenous retrieval). The specific influence of different experimental conditions on computational resources remains a topic for further investigation.

Finally, RMPFC regions that encoded intentions during occupied delays stretched along a dorsal (anterior dorsomedial PFC, BA 10) to ventral (BA 11) gradient. Previous multivariate (Haynes et al., 2007; Soon et al., 2008; Momennejad and Haynes, 2012) (Fig. 5) and univariate (Benoit et al., 2012) findings suggest that more controlled, abstract, or stimulus-independent processing recruits more dorsal RMPFC regions whereas lower control demand, low abstraction, or stimulus-oriented processing recruits more ventral RMPFC. Thus, the most rostral part of the medial PFC may itself display a dorsal-ventral division that corresponds with levels of control and the availability of computational resources. However, the specific role of the dorsal-ventral gradient of the medial PFC is yet to be systematically studied.

\section{The role of the RLPFC}

Here we show that the rostrolateral PFC (RLPFC) plays a general role in encoding future intentions with abstract task sets that does not depend on the task load during the delay. During both delay types, future task sets could be decoded from RLPFC (Fig. 3; Table 1). This finding confirms previous decoding evidence that RLPFC encodes future intentions (Haynes et al., 2007; Soon et al., 2008; Momennejad and Haynes, 2012). Consistently, lesion studies demonstrate that damage to RLPFC impairs multitasking, PM, and hierarchical planned action (Burgess, 2000; Volle et al., 2011). Of the five decoding studies of delayed intentions, including the present study, one study did not find RLPFC encoding of delayed intentions (Gilbert, 2011). Studies that found RLPFC used abstract tasks and complex S-R mappings, whereas the classification in Gilbert (2011) concerned the anticipation of specific visual cues and specific button presses rather than either an abstract task set or stimulus-independent processing. Thus, one explanation could be that tasks with more abstract rules recruit more rostral regions of the lateral PFC. Consistently, it has been suggested that progressively more rostral regions of the lateral PFC are recruited with increasing abstraction, temporal, and hierarchical organization of planned action (Koechlin et al., 2003; Sakai and Passingham, 2003; Badre and D'Esposito, 2007, '; Botvinick, 2007, 2008; Christoff et al., 2009). Together, RLPFC may be generally recruited to encode future intentions as long as the future task's rules are sufficiently abstract (Christoff et al., 2009; Momennejad and Haynes, 2012, Reverberi et al., 2012).

In conclusion, here we directly investigated computational processes that encode delayed intentions depending on the task load during the delay (i.e., over occupied and task-free delays). We found that the same future task set was encoded as follows: (1) in RLPFC during both delays, (2) in RMPFC over occupied delays, and (3) in more caudal regions, such as the ACC, the SMA, and the precuneus during task-free delays. Our findings highlight the role of RMPFC as a key computational process involved in PM. These findings can inform future brain computer interface research as well as research into PM deficit and rehabilitation after damage to PFC subregions.

\section{References}

Badre D (2008) Cognitive control, hierarchy, and the rostro-caudal organization of the frontal lobes. Trends Cogn Sci 12:193-200. CrossRef Medline

Badre D, D’Esposito M (2007) Functional magnetic resonance imaging evidence for a hierarchical organization of the prefrontal cortex. J Cogn Neurosci 19:2082-2099. CrossRef Medline

Badre D, D'Esposito M (2009) Is the rostro-caudal axis of the frontal lobe hierarchical? Nat Rev Neurosci 10:659-669. CrossRef Medline

Benoit RG, Gilbert SJ, Frith CD, Burgess PW (2012) Rostral prefrontal cortex and the focus of attention in prospective memory. Cereb Cortex 22: 1876-1886. CrossRef Medline

Bode S, Haynes JD (2009) Decoding sequential stages of task preparation in the human brain. Neuroimage 45:606-613. CrossRef Medline

Botvinick MM (2007) Multilevel structure in behavior and in the brain: a model of Fuster's hierarchy. Philos Trans R Soc B Biol Sci 362:1615-1626. CrossRef Medline

Botvinick MM (2008) Hierarchical models of behavior and prefrontal function. Trends Cogn Sci 12:201-208. CrossRef Medline

Brandimonte MA, Einstein GO, McDaniel MA (1996) Prospective memory: theory and applications. Hillsdale, NJ: Erlbaum.

Brass M, Haggard P (2008) The what, when, whether model of intentional action. Neuroscientist 14:319-325. CrossRef Medline

Braver TS, Bongiolatti SR (2002) The role of frontopolar cortex in subgoal processing during working memory. Neuroimage 15:523-536. CrossRef Medline

Burgess PW (2000) Strategy application disorder: the role of the frontal lobes in human multitasking. Psychol Res 63:279-288. CrossRef Medline

Burgess PW, Veitch E, de Lacy Costello A, Shallice T (2000) The cognitive and neuroanatomical correlates of multitasking. Neuropsychologia 38 : 848-863. CrossRef Medline

Burgess PW, Scott SK, Frith CD (2003) The role of the rostral frontal cortex (area 10) in prospective memory: a lateral versus medial dissociation. Neuropsychologia 41:906-918. CrossRef Medline

Burgess PW, Gonen-Yaacovi G, Volle E (2011) Functional neuroimaging studies of prospective memory: what have we learnt so far? Neuropsychologia 49:2246-2257. CrossRef Medline

Christoff K, Keramatian K, Gordon AM, Smith R, Mädler B (2009) Prefrontal organization of cognitive control according to levels of abstraction. Brain Res 1286:94-105. CrossRef Medline

Dismukes RK (2008) Prospective memory in aviation and everyday settings. In: Prospective memory: cognitive, neuroscience, developmental, and applied perspectives (Kliegel M, McDaniel MA, Einstein GO, eds), pp 411428. New York: Erlbaum/Taylor and Francis.

Ellis JA (1996) Prospective memory or the realization of delayed intentions: a conceptual framework for research. In: Prospective memory: theory and applications (Brandimonte MA, Einstein GO, McDaniel MA, eds), pp 1-22. Hillsdale, NJ: Erlbaum.

Friston KJ, Holmes AP, Worsley KJ, Poline JP, Frith CD, Frackowiak RSJ (1994) Statistical parametric maps in functional imaging: a general linear approach. Hum Brain Mapp 2:189-210. CrossRef

Friston KJ, Holmes A, Poline JB, Price CJ, Frith CD (1996) Detecting activations in PET and $\mathrm{AMRI}$ : levels of inference and power. Neuroimage 4:223-235. CrossRef Medline

Fuster JM (2000) Executive frontal functions. Exp Brain Res 133:66-70. CrossRef Medline

Gilbert SJ (2011) Decoding the content of delayed intentions. J Neurosci 31:2888-2894. CrossRef Medline

Gilbert SJ, Frith CD, Burgess PW (2005) Involvement of rostral prefrontal cortex in selection between stimulus-oriented and stimulus-independent thought. Eur J Neurosci 21:1423-1431. CrossRef Medline 
Gilbert SJ, Spengler S, Simons JS, Frith CD, Burgess PW (2006) Differential functions of lateral and medial rostral prefrontal cortex (area 10) revealed by brain-behavior associations. Cereb Cortex 16:1783-1789. CrossRef Medline

Gilbert SJ, Gollwitzer PM, Cohen AL, Burgess PW, Oettingen G (2009) Separable brain systems supporting cued versus self-initiated realization of delayed intentions. J Exp Psychol Learn Mem Cogn 35:905-915. CrossRef Medline

Haynes JD, Rees G (2006) Decoding mental states from brain activity in humans. Nat Rev Neurosci 7:523-534. CrossRef Medline

Haynes JD, Sakai K, Rees G, Gilbert S, Frith C, Passingham RE (2007) Reading hidden intentions in the human brain. Curr Biol 17:323-328. CrossRef Medline

Henson RNA (2004) Analysis of fMRI time series. In: Human brain function, Ed 2 (Frackowiak RSJ, Ashburner JT, Penny WD, Zeki S, Friston KJ, Frith CD, Dolan RJ, Price CJ, eds), pp 793-822. London: Academic.

Henson RNA, Rugg MD, Friston KJ (2001) The choice of basis functions in event-related fMRI. Neuroimage 13:149. CrossRef

Isoda M, Hikosaka O (2007) Switching from automatic to controlled action by monkey medial frontal cortex. Nat Neurosci 10:240-248. CrossRef Medline

Kliegel M, Martin M (2003) Prospective memory research: why is it relevant? Int J Psychol 38:193-194. CrossRef

Koechlin E (2011) Frontal pole function: what is specifically human? Trends Cogn Sci 15:241. CrossRef Medline

Koechlin E, Hyafil A (2007) Anterior prefrontal function and the limits of human decision making. Science 318:594-598. CrossRef Medline

Koechlin E, Basso G, Pietrini P, Panzer S, Grafman J (1999) The role of the anterior prefrontal cortex in human cognition. Nature 399:148-151. CrossRef Medline

Koechlin E, Ody C, Kouneiher F (2003) The architecture of cognitive control in the human prefrontal cortex. Science 302:1181-1185. CrossRef Medline

Kriegeskorte N, Goebel R, Bandettini P (2006) Information-based functional brain mapping. Proc Natl Acad Sci U S A 103:3863-3868. CrossRef Medline

McFarland CP, Glisky EL (2009) Frontal lobe involvement in a task of timebased prospective memory. Neuropsychologia 47:1660-1669. CrossRef Medline

Miller EK, Cohen JD (2001) An integrative theory of prefrontal cortex function. Annu Rev Neurosci 24:167-202. CrossRef Medline

Momennejad I, Haynes JD (2012) Human anterior prefrontal cortex encodes the 'what' and 'when' of future intentions. Neuroimage 61:139148. CrossRef Medline

Nachev P, Rees G, Parton A, Kennard C, Husain M (2005) Volition and conflict in human medial frontal cortex. Curr Biol 15:122-128. CrossRef Medline

Okuda J, Fujii T, Yamadori A, Kawashima R, Tsukiura T, Fukatsu R, Suzuki K, Ito M, Fukuda H (1998) Participation of the prefrontal cortices in prospective memory: evidence from a PET study in humans. Neurosci Lett 253:127-130. CrossRef Medline

Okuda J, Fujii T, Ohtake H, Tsukiura T, Yamadori A, Frith CD, Burgess PW (2007) Differential involvement of regions of rostral prefrontal cortex (Brodmann area 10) in time- and event-based prospective memory. Int J Psychophysiol 64:233-246. CrossRef Medline

Ramnani N, Owen AM (2004) Anterior prefrontal cortex: insights into function from anatomy and neuroimaging. Nat Rev Neurosci 5:184-194. CrossRef Medline

Reverberi C, Görgen K, Haynes JD (2012) Compositionality of rule representations in human prefrontal cortex. Cereb Cortex 22:1237-1246. CrossRef Medline

Reynolds JR, West R, Braver T (2009) Distinct neural circuits support transient and sustained processes in prospective memory and working memory. Cereb Cortex 19:1208-1221. CrossRef Medline

Rushworth MF, Walton ME, Kennerley SW, Bannerman DM (2004) Action sets and decisions in the medial frontal cortex. Trends Cogn Sci 8:410417. CrossRef Medline

Sakai K (2008) Task set and prefrontal cortex. Annu Rev Neurosci 31:219_ 245. CrossRef Medline

Sakai K, Passingham RE (2003) Prefrontal interactions reflect future task operations. Nat Neurosci 6:75-81. CrossRef Medline

Shenhav A, Botvinick MM, Cohen JD (2013) The expected value of control: an integrative theory of anterior cingulate cortex function. Neuron 79: 217-240. CrossRef Medline

Simons JS, Schölvinck ML, Gilbert SJ, Frith CD, Burgess PW (2006) Differential components of prospective memory? Evidence from fMRI. Neuropsychologia 44:1388-1397. CrossRef Medline

Soon CS, Brass M, Heinze HJ, Haynes JD (2008) Unconscious determinants of free decisions in the human brain. Nat Neurosci 11:543-545. CrossRef Medline

Terry WS (1988) Everyday forgetting: data from a diary study. Psychol Rep 62:299-303. CrossRef

Venkatraman V, Rosati AG, Taren AA, Huettel SA (2009) Resolving response, decision, and strategic control: evidence for a functional topography in dorsomedial prefrontal cortex. J Neurosci 29:13158-13164. CrossRef Medline

Volle E, Gonen-Yaacovi G, Costello Ade L, Gilbert SJ, Burgess PW (2011) The role of rostral prefrontal cortex in prospective memory: a voxel-based lesion study. Neuropsychologia 49:2185-2198. CrossRef Medline 